\section{Factors Affecting the Accuracy of Vitamin D Measurements}

Editor - In the recent issue of Clinical Medicine \& Research, we read with great interest the article by Black, et al. titled "24-Hour Urinary Calcium in Primary Hyperparathyroidism". ${ }^{1}$ Black and colleagues designed a retrospective review on patients undergoing a parathyroidectomy for primary hyperparathyroidism from 2000 - 2008. Data collected included age, sex, preoperative serum calcium (ionized and total), creatinine, parathyroid hormone (PTH), postoperative ionized calcium, vitamin D, and 24-hour urine calcium and creatinine. Relatively low correlations were observed for 24-hour urinary calcium levels with vitamin D $(P=0.04)$ in this study.

Vitamin D status is usually assessed by measuring the serum 25-hydroxyvitamin D [25(OH)D3]) concentration. This mainly depends on sunshine exposure, nutrition and age. There are several methods that are currently used for testing blood levels of Vitamin D, especially the level of total 25-OH Vitamin D in clinical laboratories. These methods include LC-MS (considered to be the gold standard for the Vitamin D test), HPLC, immunoassay and Vitamin D binding protein (DBP) based enzymatic assay. Several studies have demonstrated that results given by different methods are not comparable $^{2,3}$ although a recent DEQAS survey shows that most major methods give results within about $10 \%$ of the consensus mean. Methods could be influenced by several factors, including sample throughput, expertise of staff and sample origin. In this study, it was not mentioned whether measurement of 25(OH)D from 2000-2008 has been studied by the same method and same device ${ }^{2}$.

According to seasonal changes in Vitamin D levels, all samples must be collected in one season.

Some drugs can affect 25(OH)D levels, for example phenobarbital, isoniazid, corticosteroid. Patients using these drugs should be excluded from the study ${ }^{3}$.

UV-B radiation does not penetrate glass, so exposure to sunshine indoors through a window does not produce $25(\mathrm{OH})$ D4. The environmental conditions of the subjects in this study should be presented.

Older adults have a reduce level of 7-dehydrocholesterol, so they cannot synthesize $25(\mathrm{OH}) \mathrm{D}$ as well. Also their kidneys are less able to produce the active hormone, 1,25 dihydro Vitamin D3. In this study, ages of participants were ranging from 25 to 85 (median 63 years).

Melanin in the darker skin reduces the ability to produce $25(\mathrm{OH}) \mathrm{D}$ from sunlight exposure, because it absorbs the sunlight ${ }^{4}$. The frequency of dark skinned subjects should be mentioned in the study.

Vitamin D is fat-soluble; it is sequestered in the fat not allowing it to circulate. Also those who have obesity cannot absorb Vitamin D as readily ${ }^{5}$.

As a result, it would be useful if the authors could offer some more data fixed for major confounders including subjects' methods of measurements from 2000-2008, obesity, sunlight exposure, and the frequency of dark skin patients. This could provide the readers of the journal clearer information.

\section{References}

1. Black, CE, Berg, RL, Urquhart, AC. 24-hour urinary calcium in primary hyperparathyroidism. Clin Med Res 2013;11: 219-225.

2. Carter, GD. Accuracy of 25-hydroxyvitamin D assays: confronting the issues. Curr Drug Targets 2011;12:19-28.

3. Holick MF. Vitamin D: A millenium perspective. J Cell Biochem 2003;88:296-307.

4. Holick MF. Sunlight and vitamin D for bone health and prevention of autoimmune diseases, cancers, and cardiovascular disease. Am J Clin Nutr 2004;80:1678S-1688S.

5. Rajakumar K, Fernstrom JD, Holick MF, Janosky JE, Greenspan SL. Vitamin D status and response to Vitamin D(3) in obese vs. non-obese African American children. Obesity (Silver Spring) 2008;16:90-95.

Fevzi Nuri Aydin

Department of Biochemistry Sirnak Military Hospital

Sirnak, Turkey

E-mail:fevzinuriaydin@gmail.com

Ibrahim Aydin Department of Biochemistry Sarikamis Military Hospital Sarikamis Kars, Turkey

Mehmet Agilli Department of Biochemistry Agri Military Hospital Agri, Turkey

Received: February 26, 2014

Accepted: March 25, 2014

doi: $10.3|2| / \mathrm{cmr} .20|4.123|$ 